$(\mathrm{n}=22)$ and community workers working with Pakistani youth $(\mathrm{n}=8)$. Young Pakistanis were purposively selected across community settings: aged 16-25, born in UK and/or had been through UK secondary school. Interviews aimed to establish key life, social and cultural issues, with focus on relationship type and formation and perceived need/support. Interviews were recorded and transcribed verbatim. Analysis was thematic using "Framework" approach.

Results Many themes emerged which have a bearing on, and shape young Pakistanis' experiences of, relationships. Most experience parental restrictions on socialising and mixing with the opposite sex. For girls, behaviour is further monitored by older brothers and for both sexes "community policing" is an extension of this. However, young people have developed creative strategies to circumvent these restrictions and despite faith and cultural norms, relationships do take place, primarily in secrecy. This presents what are described as "conflicting pressures", "double worlds", and "multiple realities", which young people negotiate and move between, balancing different value systems. There were striking gender differences in perceptions and types of relationships and intimacy. For young women the ideal was a relationship for marriage. However, many described partners as "bad boys" and "gangster types" and some relationships were considered pressurised and "unhealthy". The young men made distinctions between girls deemed "wifey material" and those for casual relationships. Older boys $(18+)$ were sexually active with partners from a range of ethnic backgrounds. Condom use was inconsistent. Though not sexually active, some of the other young people had experienced mutual touching and/or oral sex. Few had good sexual health knowledge and would not know where to access help.

Conclusions The secrecy within which young Pakistanis have relationships and the pressures and gender roles they negotiate mean that many may not receive the support they need. This has implications for the delivery of appropriate preventative and curative sexual health services.

\section{VARIATIONS IN THE USE OF PUBLICLY FUNDED ORAL CARE IN NORTHERN IRELAND: RESULTS FROM AN ANALYSIS OF ADOLESCENTS IN THE NORTHERN IRELAND LONGITUDINAL STUDY}

doi:10.1136/jech.2010.120956.73

${ }^{1} \mathrm{C}$ Telford, ${ }^{1} \mathrm{~L}$ Murray, ${ }^{2} \mathrm{C}$ 0'Neill. ${ }^{1}$ Centre for Public Health, Queen's University Belfast; ${ }^{2} \mathrm{NUI}$ Galway

Background A socio-economic gradient in use of health care has been observed in a number of situations. These have been used to assess the performance of systems as well as frame discussion on system design. Examination of patterns at an aggregate level may mask important differences between types of care that could lead to different policy advice.

Aims To identify whether differences in registration and use related to socio-demographic characteristics exist in respect of publicly funded oral health care in Northern Ireland and if so to identify the nature of differences in care.

Methods NHS reimbursement data were linked to census and vital statistics data within the Northern Ireland Longitudinal Study. Data cover $28 \%$ of the population in Northern Ireland and in this study cover a period from 2003 to 2008. Data for individuals aged 11 or 12 in April 2003 that include registration status, reimbursement on a per item basis, gender, community background, siblings and also the social class and education of household reference person (HRP) were extracted. A series of multivariate analyses were used to examine the relationship between registration and use of care as a function of socio-demographic characteristics.
Results A clear socio-economic gradient was evident in respect of registration status. Adolescents whose HRP was long term unemployed or never worked were registered for 6 months (from a maximum of 54) less and consumed $8.3 \%$ less expenditure than those whose HRP was professional. While those from lower social backgrounds consumed $24.4 \%$ less expenditure on orthodontic services, with respect to extractions and conservative treatment, adolescents whose HRP was long term unemployed or never worked consumed $35.6 \%$ and $25.8 \%$, respectively, more expenditure than those whose HRP was professional, other variables controlled for.

Conclusions A publicly funded demand led service can produce a pattern of service provision that disproportionately reflects the preferences of the affluent at the expense of the needs of the less affluent. This might be masked by analysis of data at an aggregate level. The pattern of service provision that results may from a societal perspective be neither efficient (cost effective) nor equitable. The disaggregated analysis of registration and utilisation patterns in this study highlights the potential for such distortion where public funds support demand led provision by for profit providers.

\section{WHO THINKS TEENAGERS ARE A PROBLEM? CROSS- SECTIONAL EVIDENCE ON PERCEPTIONS OF ANTI-SOCIAL BEHAVIOUR, HEALTH AND PLACE}

doi:10.1136/jech.2010.120956.74

M Egan, on behalf of the GoWell team. Medical Research Council/Chief Scientist Office Social and Public Health Sciences Unit, Glasgow, UK

Objective Perceptions of anti-social behaviour (PASB) are a policy priority and linked to numerous social harms including experience of ASB. National survey findings have associated PASB with individual and area level deprivation, potentially adding to the multiple disadvantages said to contribute to health inequalities. We ask whether PASB have independent associations with ill health compared to other individual and neighbourhood characteristics for residents of deprived inner-city neighbourhoods (Glasgow, UK).

Methods Randomly selected adult residents of 14 deprived neighbourhoods answered face-to-face structured questionnaires. A stepwise logistic regression (clustered by area) produced a multivariate model including self-rated measures of health, GP visits, psychosocial wellbeing, homes, neighbourhoods, and demographic characteristics. Perceiving teenagers hanging around to be a serious neighbourhood problem was the dependent variable.

Results 6008 adults participated (50\% response). Regular annual GP visits $(>6)$ were associated with PASB (OR 1.29; $\mathrm{p}=0.011$; $95 \% \mathrm{CI}$ 1.06 to 1.56 ), as was GP visits $(>0)$ for mental health reasons (OR 1.44; $\mathrm{p}=0.020 ; 95 \%$ CI 1.06 to 1.96$)$. PASB was inversely associated with self/collective efficacy (OR 1.25; $\mathrm{p}=0.028 ; 95 \%$ CI 1.02 to 1.53); self-esteem (OR 1.56; $\mathrm{p}<0.001 ; 95 \%$ CI 1.30 to 1.87 ); trust (OR 1.53; $\mathrm{p}<0.001 ; 95 \%$ CI 1.19 to 1.96); feeling safe (OR 1.71; $\mathrm{p}<0.001 ; 95 \%$ CI 1.41 to 2.06 ); social support (OR 1.94; $\mathrm{p}<0.001$; $95 \%$ CI 1.45 to 2.59 ); age (OR 2.33; $\mathrm{p}<0.001 ; 95 \%$ CI 1.56 to 3.50 ); living with children (OR $1.20 ; \mathrm{p}=0.001 ; 95 \%$ CI 1.06 to 1.37 ) home condition (OR 1.31; $p=0.006$; $95 \%$ CI 1.08 to 1.58 ); home security (OR 1.31; $p=0.042 ; 95 \%$ CI 1.01 to 1.71 ); neighbourhood exposure (OR 1.60; $\mathrm{p}=0.015 ; 95 \%$ CI 1.09 to 2.34 ); rating of police (OR 1.65; $\mathrm{p}<0.001 ; 95 \%$ CI 1.34 to 2.02$)$. PASB was positively associated with fuel poverty (OR 1.43; $\mathrm{p}=0.011 ; 95 \%$ CI 1.09 to 1.87 ); neighbour contacts (OR 1.60; $p=0.005 ; 95 \%$ CI 1.10 to 1.71 ); neighbourhood decline (OR 2.42; $\mathrm{p}<0.001 ; 95 \%$ CI 1.84 to 3.89); noisy environment (OR $1.23 ; p=0.042 ; 95 \%$ CI 1.01 to 1.50 ), and area type (OR 1.59 ; $\mathrm{p}<0.001 ; 95 \%$ CI 1.33 to 1.91$)$. General health and longstanding illness were not associated with PASB after adjustment $(\mathrm{p}>0.05)$.

Conclusion National surveys link PASB to socio-economic status but the deprived communities we surveyed have relatively little socio- 
economic variance. Therefore other characteristics like GP use (particularly for mental health reasons) and psychosocial wellbeing become more important in explaining PASB. Future longitudinal research is planned to explore causation-for example, do GP users worry about teenagers because illness encourages feelings of vulnerability-or does worrying about neighbourhood problems exacerbate ill health? Current findings are consistent with the view that addressing PASB be included in strategies to address health inequalities.

\section{Alcohol

075 GAMMA-GLUTAMYLTRANSFERASE AS A PREDICTOR FOR
ALCOHOL- AND NON-ALCOHOL-RELATED CANCER
INCIDENCE

doi:10.1136/jech.2010.120956.75

${ }^{1} \mathrm{~S}$ Strohmaier, ${ }^{1} \mathrm{~W}$ Borena, ${ }^{1} \mathrm{~A}$ Strasak, ${ }^{1} \mathrm{G}$ Goebel, ${ }^{1} \mathrm{M}$ Edlinger, ${ }^{2} \mathrm{G}$ Diem, ${ }^{2} \mathrm{H}$ Concin, ${ }^{3} \mathrm{C}$ Kelleher, ${ }^{1} \mathrm{H}$ Ulmer. ${ }^{1}$ Department for Medical Statistics, Informatics and Health Economics, Innsbruck Medical University, Innsbruck, Austria; ${ }^{2}$ Agency for Preventiveand Social Medicine, Bregenz, Austria; ${ }^{3}$ School of Public Health and Population Science, University College Dublin, Dublin, Republic of Ireland

Objective Recent evidence suggests that elevated levels of gammaglutamyltransferase (GGT) are associated with both incidence and mortality of cardiovascular disease and cancer. Although GGT is regarded as a marker of liver function which may in turn reflect alcohol consumption, to date, no study has investigated the relationship of GGT with cancer sites known to be alcohol-related or non-related.

Design Prospective cohort follow up and linkage study.

Participants and setting First visit measurements in 94628 adult women and 80224 men screened for metabolic risk factors as part of a standardised primary care assessment in Vorarlberg province of Austria. During a median follow-up of 13 years, a total of 5136 incident cancers were diagnosed in men and 4665 in women.

Methods Sex-specific Cox proportional hazards models, adjusted for age, body mass index and smoking were performed to estimate HRs and $95 \%$ CI per quintiles of GGT.

Results In males, the highest GGT-quintile revealed a high risk of alcohol-related cancer incidence (HR 2.20, 95\% CI 1.74 to to 2.78). The association was strongest for cancers of the liver and intrahepatic bile ducts (HR 16.50, 4.00-68.19), followed by cancers of the lip, oral cavity, pharynx and larynx (HR 3.80, 2.33-6.20), esophageal cancer (HR 2.39, 1.01-5.72) and colorectal cancer (HR $1.36,1.01-1.83)$. In females, there was a modest but significant association between GGT and alcohol-related cancers (HR 1.16, 1.02-1.32). GGT showed a significant association in breast cancer only (HR 1.19, 1.02-1.39). HRs were clearly elevated for cancers of the liver and intrahepatic bile ducts and for cancers of the lip, oral cavity, pharynx, larynx and oesophagus, however, without reaching significance due to limited number of cases. No association was seen for colorectal cancer. Additionally, elevated GGT was found to be significantly related to cancers with weak or no evidence of alcohol consumption as a risk factor. In males, there were associations with pancreatic cancer (HR 2.13, 1.01-4.56), lung cancer (HR 2.04, 1.55-2.70), bladder cancer (HR 1.76, $1.11-2.77$ ) and kidney cancer (HR 1.61, 0.92-2.82, $\mathrm{p}$ for trend $=0.009$ ). In females, the association was most pronounced in cervical cancer (HR 3.77, 1.94-7.32), followed by lung cancer (HR $1.63,1.02-2.60$ ) and endometrial cancer (HR 1.42, 0.98-2.05, p for trend $=0.013$ ).

Conclusion Although elevated GGT levels were strongly associated with incidence of alcohol-related cancers, most markedly in men, there were still effects of GGT in non-alcohol related cancer sites.
This suggests that alcohol consumption explains the relationship between GGT and cancer outcomes only in part.

\section{BINGE DRINKING IN MIDLIFE AND THE RISK OF DEVELOPING DEPRESSION DURING YEARS OF FOLLOW-UP}

doi:10.1136/jech.2010.120956.76

S Bell, A Britton, M Shipley. Department of Epidemiology and Public Health, University College London, London, UK

Objective To examine the relationship between binge drinking at baseline and the onset of new depression during 24 years of followup after adjustment for age, socio-economic status, education and marital status.

Design and setting Data from phases 1 (1985-1988) to 9 (2007-2009) of the Whitehall II prospective cohort were used.

Participants 5985 (male $=4161$, female $=1824$ ) British civil servants aged 35-55 years who were free from depression at baseline.

Comparison groups Cohort members were grouped, at phase 1, according to their self-reported usual and maximum number of alcoholic drinks consumed in a single sitting-abstainers, nonbingers (reference category) and bingers. Alcohol consumption was split into two categories and number of drinks consumed was converted to units for analysis: wine and spirits ( 1 unit per drink), and beer ( 2 units per drink). For usual drinking sessions those who reported consuming $5+$ units of wine/spirits and $10+$ units of beer were categorised as bingers, those consuming 1-4 units of wine/ spirits or 1-9 units of beer were classified as non-bingers. For maximum drinking sessions, participants were defined as bingers following the Department of Health guidelines as those consuming $8+$ or $6+$ units of alcohol for males and females respectively for both categories of consumption. Those who reported consuming no drinks were classified as abstainers in all analyses.

Main outcome measures The 30-item General Health Questionnaire (GHQ-30) was administered at all phases of data collection. The depression subscale of the GHO-30 was used to identify new cases of depression (scores of 4 or more) across all phases.

Results Adjusted HRs and 95\% CIs were estimated using Cox proportional hazard models fitted in the total cohort and stratified by gender. Usual drinking session spirit/wine bingers had an elevated risk of depression (HR 1.28, CI 1.02 to to 1.60) compared to non-bingers in the total sample. Maximum drinking session spirit/ wine bingers had a greater risk of depression in the total (HR 1.23, CI 1.04 to to 1.44 ) and male (HR 1.27, CI 1.03 to to 1.56 ) samples. There were no statistically significant effects when using beer measures as exposures or for abstainers in any alcohol measures after adjustment for confounders.

Conclusion Binge drinking on wine and spirits, but not beer, in midlife increases the risk of having a depressive episode over the course of the following 22-24 year period. Future work will examine other covariates and explore bidirectional issues in this relationship.

\section{ALCOHOL USE AMONG RUSSIAN MEN: THE ASSOCIATION BETWEEN AUDIT SCORE AND SELF- AND PROXY-REPORTED DRINKING BEHAVIOURS}

doi:10.1136/jech.2010.120956.77

S Cook, B De Stavola, D Leon. London School of Hygiene and Tropical Medicine, London, UK

Objectives Alcohol use in Russia has a number of relatively distinct features which have been shown to be strongly associated with mortality. The aim of this paper is to investigate how specific 